\title{
Hybrid LES/RANS predictions of flows and acoustics from an ultra-high-bypass-ratio serrated nozzle
}

\author{
Zhong-Nan Wang, James Tyacke and Paul Tucker
}

\begin{abstract}
In this paper, the jet flow from an industrially relevant ultra-high-bypassratio(UHBPR) serrated nozzle has been simulated in a flight stream. The methodology to tackle complex geometries and multi-disciplinary physics is demonstrated in detail and validated using a single stream jet with measurements. A reliable industrial process chain is explored. This type of complex geometry jet simulation shows great potential to replace parts of experimental tests in the near future.
\end{abstract}

\section{Introduction}

Jet noise is still one of the dominant components when an aircraft is taking off. In order to reduce the noise emitted, the first step is to predict it accurately. Aviation noise standards require the jet to be operating under forward flight conditions. Measuring jet noise in forward flight is not easy to achieve in ground test rigs. Open jet facilities are often used for this test, but generate an additional shear layer from the edge of the larger flight stream nozzle. This additional shear layer introduces extra noise and diffracts the propagation of jet acoustic waves [1, 2]. In addition to testing difficulty, the maintenance of such facilities is also expensive. Thus, the reliable numerical prediction of jet noise is clearly required for low noise aeroengine design.

Large-eddy simulation is a vital method to capture the unsteady coherent flow structures in jets and is increasingly being used to predict the flow and acoustics of propulsive jets $[3,4,5]$. As the effects of the forward flight stream and wing installation are considered, the boundary layer development on the engine nacelle

Zhong-Nan Wang

Department of Engineering, University of Cambridge, e-mail: znw22@cam.ac.uk

James Tyacke

Department of Engineering, University of Cambridge, e-mail: jct53@cam.ac.uk

Paul Tucker

Department of Engineering, University of Cambridge, e-mail: pgt23@cam.ac.uk 
and the wing/flap becomes important to the turbulence and acoustics generated. To keep the computational cost affordable at industrially relevant Reynolds numbers, RANS zones are required near the nozzle and the wing to predict turbulent boundary layer profiles and circumvent nonphysical separations [6]. This type of highly complex geometry jet simulation will replace rig testing in the near future and we are seeking to explore a reliable industrial process chain.

In this paper, the jet flow from an industrial relevant UHBPR engine has been simulated in forward flight. Serrations are attached to the nacelle to enhance the shear layer mixing and hence reduce jet noise. Hybrid structured/unstructured mesh and RANS/LES modelling are demonstrated for the serrated nozzle jet. The results are shown in terms of near-field flows and far-field acoustics.

\section{Methodology}

The methodology used for the jet noise prediction is demonstrated in this section. It includes a low-dissipation numerical scheme, hybrid LES/RANS turbulence modelling and far field acoustics prediction.

\subsection{Numerical Methods}

Eddy resolving simulations require the majority of flow energy (around 90 percent) to be directly resolved instead of modelled. Thus, more demand is put on the numerical method to resolve the turbulence accurately. The numerical method should ideally be non-dissipative and non-linearly stable. Too much numerical dissipation can delay transition processes, smear turbulence eddies, degrade turbulent spectra and even laminarize turbulent flows.

The kinetic energy preserving (KEP) scheme is used in our simulation [7]. The kinetic energy preserving property is built in the numerical discretization to ensure the non-linear numerical stability without any artificial dissipation. The convective flux can be written as

$$
F_{n}^{I}=F_{n}^{K E P}-\frac{1}{2} \varepsilon|A|\left[L\left(U_{R}\right)-L\left(U_{L}\right)\right]
$$

where, $F_{n}$ is the surface normal flux vector, $U$ is the conservative variable vector, $\left|A_{n}\right|=\left(\frac{\partial F}{\partial U}\right)_{n}$ is absolute Jacobian matrix, $L$ is the pseudo Laplacian operator, $($.$) is$ the averaging operator on two nodes of the edge, the subscript $L$ and $R$ is the right and left nodes on an edge across the control volume face. The parameter $\varepsilon$ controls artificial dissipation in the simulation. In the LES zone, $\varepsilon$ is kept at a low level and is increased to develop a sponge region towards the boundary.

The turbulence cascade is largely governed by the kinetic energy transfer process from large scales to small scales. The KEP scheme is a numerical method 
that guarantees this process globally in the simulation. Homogeneous isotropic decaying turbulence is tested using KEP scheme, shown in Fig. 1. The KEP scheme also displays lower sensitivity to cell type compared to the upwinding Roe Scheme. Hence, it seems more suitable for the hybrid structured-unstructured meshes used. This forms a solid foundation for eddy resolving simulation.

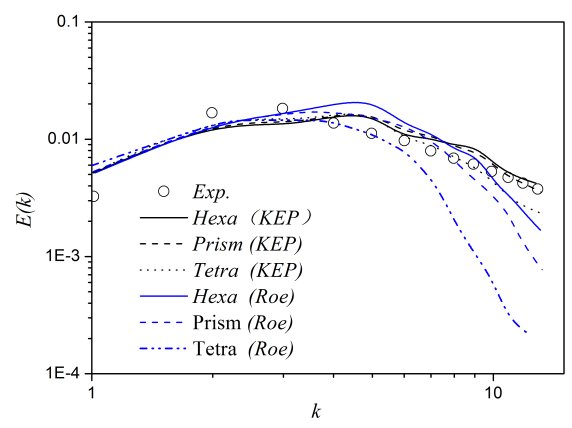

Fig. 1: Validation of homogeneous isotropic decaying turbulence

Dual time integration is employed for time marching. This uses the three-level backward Euler scheme to discretize the physical time derivatives, providing a temporal scheme of $2^{\text {nd }}$ order. The inner pseudo iterations are performed using 5 -stage Runge-Kutta scheme. Normally, ten inner pseudo iterations are used to drive the residual down by around two orders of magnitude. As the outer time loop is discretized implicitly, larger physical time steps are attainable in RANS layer, to better align with the LES zone, thus increasing the computational efficiency compared to purely explicit time marching.

\subsection{Hybrid LES/RANS Modelling}

To avoid the cost of directly resolving attached boundary layers on the nozzle of propulsive jets, a RANS layer is used near the wall to model the inner layer. In the simulations, the RANS layer is blended with the LES zone based on a modified wall distance [8]. For the LES zone, the non-linear subgrid stress model, mixed LANS- $\alpha$ model [9], is used to treat small turbulence scales. The mixed linear and non-linear model has been verified to be effective for jet flows [10]. The formula of this hybrid LES/RANS strategy can be written as

$$
\tau_{i j}^{m o d e l}=\bar{L}+\overline{N L}
$$


where, $\bar{L}$ and $\overline{N L}$ represent the linear and non-linear part, respectively, of the modelled turbulence stress. The blending is performed in these two terms between the SA RANS model [11] and mixed LANS- $\alpha$ SGS model [10]. The blended linear and nonlinear stress terms are

$$
\begin{array}{r}
\bar{L}=\frac{\tau_{k k} \delta_{i j}}{3}+2\left[f \cdot \mu_{S G S}+(1-f) \cdot \mu_{R A N S}\right] S_{i j} \\
\overline{N L}=\rho C_{\alpha} \Delta^{2} f\left(\frac{\partial u_{i}}{\partial x_{l}} \frac{\partial u_{l}}{\partial u_{j}}+\frac{\partial u_{i}}{\partial x_{l}} \frac{\partial u_{j}}{\partial u_{l}}+\frac{\partial u_{l}}{\partial x_{i}} \frac{\partial u_{l}}{\partial u_{j}}\right)
\end{array}
$$

where, $S_{i j}=0.5\left(\partial u_{i} / \partial x_{j}+\partial u j / \partial x_{i}\right)$ is the strain rate tensor, $\Delta=v_{o l} l^{1 / 3}$ is the filter length. The blending function $f$ is based on the wall distance:

$$
f=\min \left[\max \left(\frac{d_{\text {wall }}-(1-\beta) d_{R A N S}}{\beta d_{\text {RANS }}}, 0\right), 1\right]
$$

The wall distance $d_{\text {wall }}$ and the RANS layer thickness $d_{R A N S}$ are used to perform a linear blending in a small region, the factor $\beta$ defines the size of RANS-to-LES transitional zone. The RANS layer thickness can be specified locally on wall boundary patches to reflect different Reynolds numbers in different streams. The bending function and resulting SA variables are shown in Fig. 2. The RANS layer on the nacelle is thicker than that on the other wall boundaries, to match the incoming boundary layer thickness in the experiment.

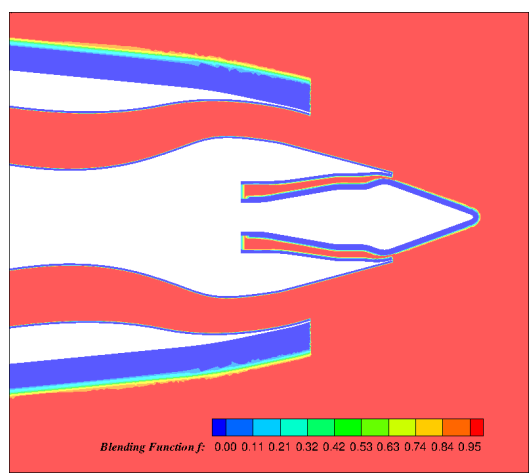

(a) Blending function $f$

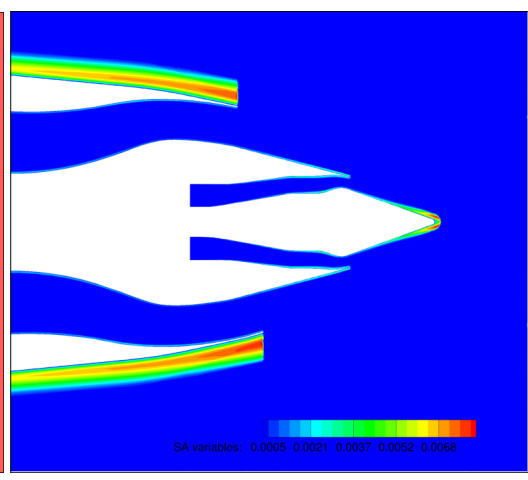

(b) SA variables

Fig. 2: Blending function and SA variables for hybird RANS/LES 


\subsection{Ffowcs Williams-Hawkings(FW-H) Method}

Acoustic pressure waves are produced by turbulence-generated sound sources in the near field and propagate to the far field. In this paper, the sound source in the nearfield is captured by the hybrid RANS/LES simulation. Instead of directly solving the acoustic pressure propagation, FW-H method is used to predict the far-field sound based on the unsteady near-field flow. The FW-H method is based on the Lighthill analogy and requires integration over the near-field probe surface for monopole and dipole sources and over the volume out of the probe surface for quadrupole sources [12]. Near-field time series are recorded of the primitive variables on the permeable FW-H surface. We usually include all sound sources within the FW-H surface so the quadrupole volume integration can be neglected, which can result in substantial savings in storage and computation. The formula used here is the convective form, which is referred as the formula 1c [13] and accounts for the uniform flight stream directly.

$$
4 \pi \tilde{p^{\prime}}(x, t)=\int_{S}\left[\left(1-M_{0} \tilde{R_{1}}\right) \frac{\dot{Q}_{i} n_{i}}{R^{*}}-U_{0} \frac{\tilde{R}_{1}^{*} Q_{i} n_{i}}{R^{* 2}}\right] d S+\int_{S}\left[\frac{\dot{L_{i j}} n_{j} \tilde{R}_{i}}{c_{0} R^{*}}+\frac{L_{i j} n_{j} \tilde{R}_{i}^{*}}{R^{* 2}}\right] d S
$$

where, $x$ is observer location and $y$ is source location. $U_{0 j}$ is the flight stream velocity in the $x_{j}$-direction. The mass flux is $Q_{i}=\rho U_{i}-\rho_{0} U_{0 i}$, the momentum flux is $L_{i j}=\rho u_{i}^{\prime}\left(u_{j}^{\prime}+U_{0 j}\right)+p^{\prime} \delta_{i j}$. The time derivatives is expressed by $(\dot{*})=\partial(*) / \partial t$, while the spatial derivative is expressed by $(\tilde{*})_{i}=\partial(*) / \partial x_{i}$. The acoustic distance $R^{*}$ is defined as

$$
\begin{array}{r}
R^{*}=\sqrt{\left(x_{1}-y_{1}\right)^{2}+\beta\left[\left(x_{2}-y_{2}\right)^{2}+\left(x_{3}-y_{3}\right)^{2}\right]} \\
\beta=\sqrt{1-M_{0}^{2}} \\
R=\frac{-M_{0}\left(x_{1}-y_{1}\right)+R^{*}}{\beta^{2}}
\end{array}
$$

In our simulation, five sets of FW-H surfaces have been placed around the jet plume with maximum outer diameter from 2.5D to 4.5D. Each of FW-H surface has 21 end disks from 20D to 30D with an interval of $0.5 \mathrm{D}$. A schematics of FW-H surface placement is shown in Fig. 3, the near field pressure is contoured.

\section{Hybrid Meshing Strategy for Chevron Geometries}

Chevrons are included at the nozzle exit. In addition to the geometric complexity, the shear layer coming from the chevrons are non-axisymmetric and very threedimensional. The optimal mesh design requires the grid to follow the shear layer 


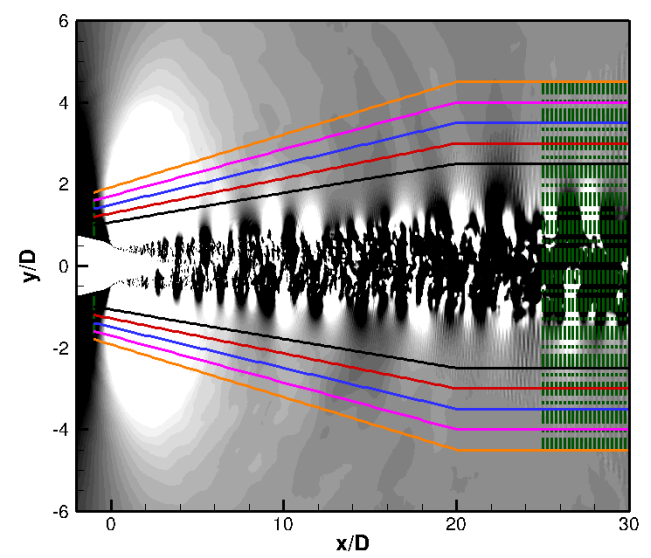

Fig. 3: FW-H surfaces placement in the near field

and to be fine enough in the circumferential direction near the nozzle exit to resolve small scale instabilities and then gradually get coarser azimuthally downstream when the scales grow larger. From these aspects, it is extremely difficult and timeconsuming to mesh this complex geometry using a fully structured grid. In our simulation, a modular embedded mesh is employed using hybrid structured/unstructured mesh to represent the chevrons. This makes the meshing process much more flexible and easier to keep the mesh of high quality in the LES region. The concept of this hybrid mesh is demonstrated in Fig. 4.

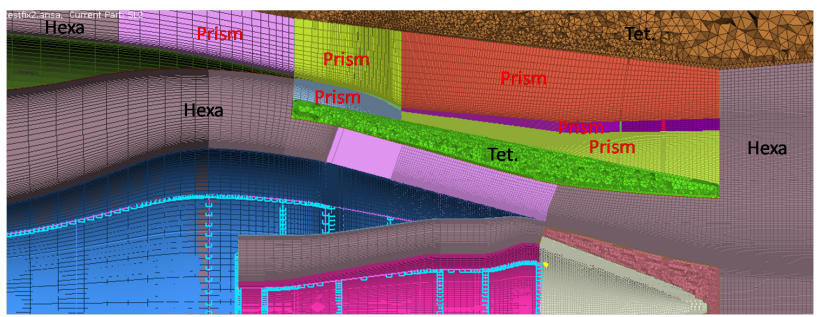

(a) Hybrid mesh topology

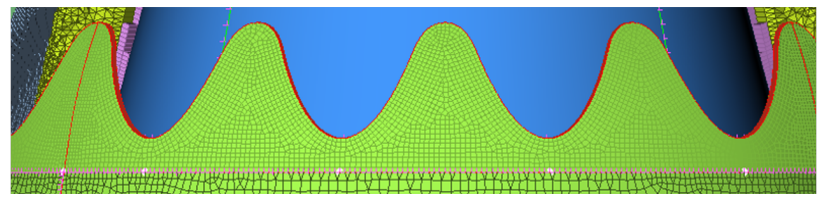

(b) Quad dominant surface meshes on the chevrons

Fig. 4: Illustration of hybrid mesh strategy 
First, the structured hexahedral mesh, which is most suitable for LES, is used in the jet plume similar to that used for the round nozzle [14]. The structured mesh aligns with the flow and reduces grid cell count compared to tetrahedral mesh. On the outside of the jet plume, the structured mesh is interfaced with isotropic tetrahedral mesh $[14,15]$. The isotropic tetrahedral mesh is used in the near-field acoustic region to better resolve the sound wave propagation. This allows local control of resolution to target acoustic frequencies and also aids the introduction of further complex geometry such as a wing and flap. To mesh the chevron, prism layers are used both on the chevrons and along the shear layer. The quad dominant surface meshes are generated on the chevrons and shear layer stream-surfaces for prism meshes to grow from, shown in Fig. 4(b). The quad dominant dominant surface mesh results in a hexahedral-dominant volume mesh, which is usually preferable numerically. This type of mesh strategy can also facilitate the grid resolution variation in the azimuthal direction.

The second aspect is to follow non-axisymetric chevron shear layers in the meshing process. This better utilizes the dense mesh from the nozzle boundary layer to resolve the large gradient across the shear layer. To estimate the shear layer location before doing LES, RANS has been preformed to use as a indicator of the shear layer trajectory with a coarser mesh. A parabolic function is designed to detect the shear layer trajectory.

$$
S L_{\text {dectection }}=\frac{U(\mathbf{x})-U_{\text {flight }}}{U_{\text {bypass }}-U_{\text {flight }}} \cdot\left(1-\frac{U(\mathbf{x})-U_{\text {flight }}}{U_{\text {bypass }}-U_{\text {flight }}}\right)
$$

where, $U(\mathbf{x})$ is the axial velocity at the position $\mathbf{x}, U_{\text {flight }}$ is the flight stream axial velocity, $U_{\text {bypass }}$ is the maximum axial velocity in the bypass duct.

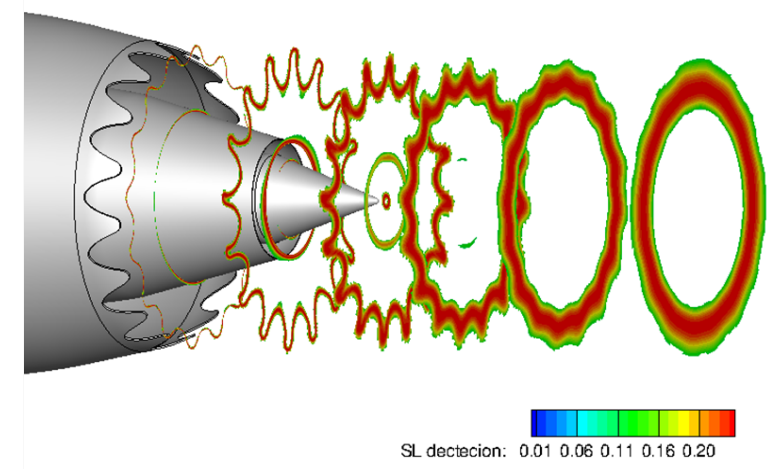

Fig. 5: Shear layer detection in chevron jets

The function highlights the shear layer by the value from 0 to 0.25 , Figure 5 shows the detected shear layer emanating from the chevrons. It varies periodically in azimuthal directions. Although RANS is inaccurate to predict the jet spreading 
rate, the initial tests revealed good agreement between RANS and LTES on the shear layer trajectory within $\mathrm{x}=2 \mathrm{D}$. The RANS informed mesh and final LES flow field are shown in Fig. 6. The grid matches with the shear layer very well, which makes the best use of the mesh to resolve the sharp gradient in the shear layers. Further mesh movement results in highly distorted cells.

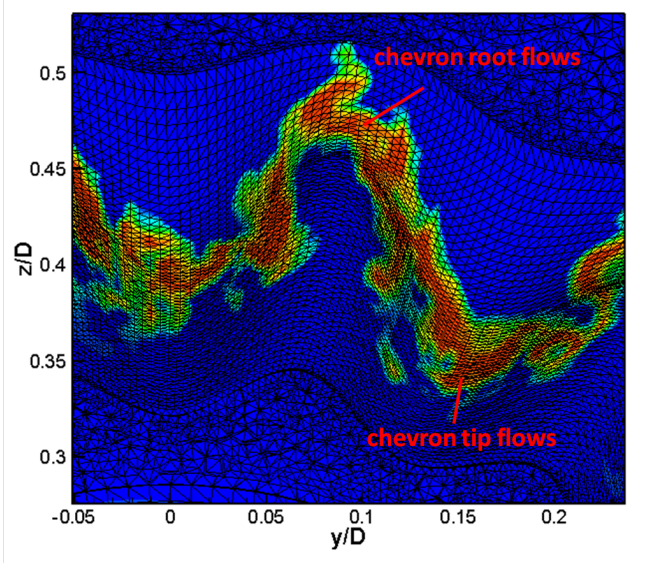

Fig. 6: Shear layer detection in chevron jets

\section{Results and Discussions}

In this section, the methodology, shown in the previous sections, is first validated on a single stream hot jet for both flows and farfield sound. Then the results of an UHBPR engine jet are presented in terms of near-field turbulence and far-field acoustics.

\subsection{Validation on a single stream hot jet}

The single stream hot jet is first computed using the described methodology. The jet is operating at $R e=4 \times 10^{5}$ and $M a=0.87$ with temperature ratio of 2.7. The near-field flows are compared with J. Bridges' experimental data [16] which is obtained on a different nozzle at similar operating conditions. Figure 7 shows the time-averaged axial velocity and turbulence intensity along the jet centreline. The simulation shows reasonable agreement with experiment measurement. It is worth 
noting that the experiment is running at slightly higher $M a=0.9$ and with no flow contraction out of the nozzle so the jet potential core should be a little longer.

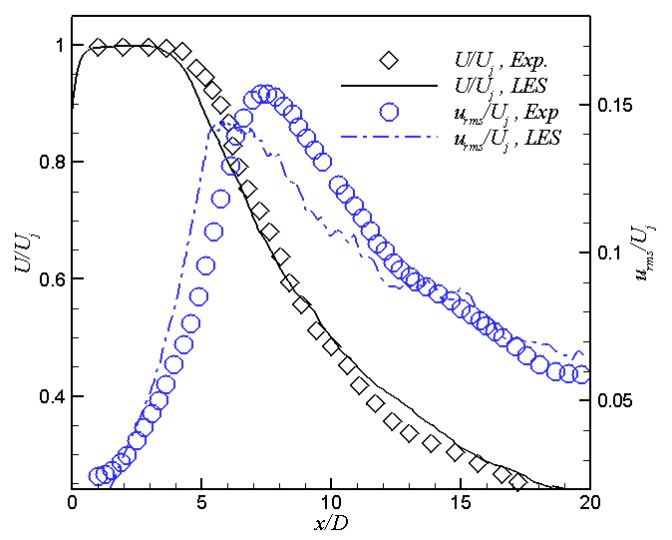

Fig. 7: Time-averaged axial velocity and turbulence intensity along the centreline

For the farfield, the sound is predicted using the FW-H method. Another experiment is done at the same condition as that in the simulation to measure the far-field acoustics. The overall sound pressure level (OASPL) and acoustic pressure spectra is shown in Fig. 8. The OASPL agrees with the experiment measurement very well within $1 \mathrm{~dB}$ and the cut-off Strouhal number is up to 5 in the sound pressure spectra. The results of the single stream jet proves that our methodology is reliable and accurate for propulsive jets and their sound prediction.

\subsection{Application to an UHBPR serrated nozzle jet}

\subsubsection{Turbulent flows in the near field}

Following validation, the UHBPR engine jet has been simulated using the same methodology. The number of cells is around 60 million with 40 azimuthal points per chevron near the nozzle, where the structures are very small, and 10 points per chevron further downstream, where the flow structure becomes axisymmetric and larger than one chevron azimuthally. Figure 9 shows the resolved flow structures from the chevron nozzle. The structures are coloured by radial velocity. Near the nozzle, the flow from the chevron root is moving outwards while the flow from the chevron tip is moving inwards. On top of the streamwise shear, this generates extra shear in the radial direction, which leads to streamwise vorticity. This is the 


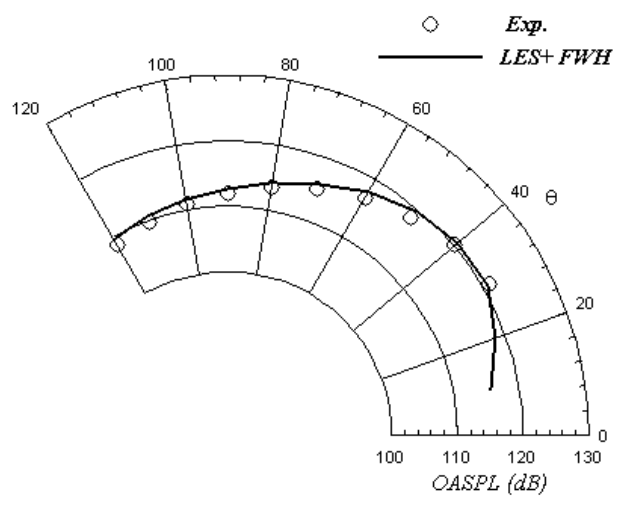

(a) Overall sound pressure level(OASPL)

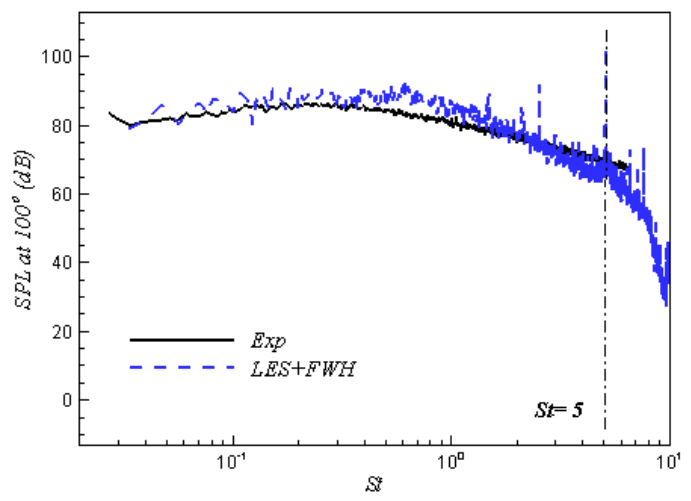

(b) Acoustic pressure spectra at $100^{\circ}$

Fig. 8: Far-field sound prediction of the single stream hot jet

principle that the chevron can enhance the mixing and shows potential to reduce the radiated noise. Figure 9 also shows that the scales are growing larger when the flow is moving away from the nozzle, so less grid points are needed to resolve it.

The shear layer non-axisymmetricity is one of the main features in the chevron nozzle jet. Figure 10(a) overviews the shear layer development from the chevrons. The flow is azimuthally periodic when exiting the chevrons, then becomes round by mixing further downstream. The variation in radial position of the shear layer downstream of the chevron root and tip is shown in Fig. 10(b). The development of shear flow can be classified by three phases: In the first phase, non-axisymmetric instability is introduced by the chevrons and develops over the first $0.5 \mathrm{D}$; Then the mixing increases and balances with the azimuthal mean flow variation, so a nonaxisymmetric level is maintained over the next $0.5 \mathrm{D}$; After this, the mixing prevails 


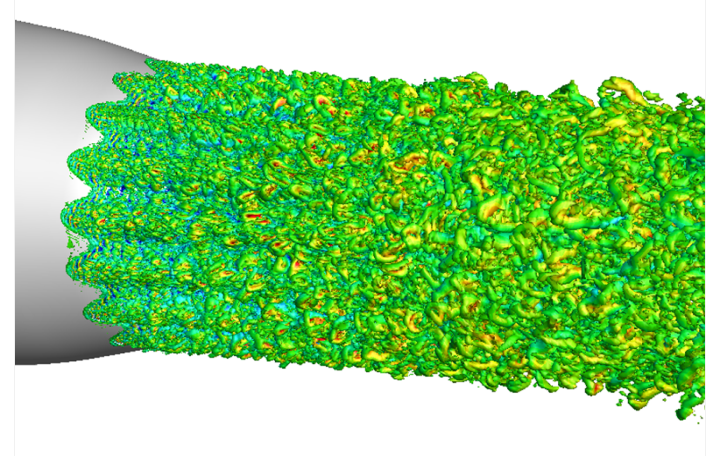

Fig. 9: Q-criterion of the ultra-bypass ratio chevron jet

and the azimuthal variation decays very fast, hence the jet becomes axisymmetric at around $\mathrm{x}=4 \mathrm{D}$.

The development of jet plume turbulence is shown in Fig. 11. The turbulence develops quickly from the outer lip due to the strong instability introduced by chevrons. For the inner shear layer, the turbulence disturbance is delayed. There are two potential causes: first, the core flow is heated to a higher temperature that increases the fluid viscosity; Small velocity difference between the bypass and the core also gives weaker K-H instability. Both of these two factors contribute to the delayed transition process. The outer shear layer finally meets with the inner at around $\mathrm{x}=4 \mathrm{D}$ and triggers a bypass-type transition of the inner. As it develops further downstream, both shear layers merge at the centre line at around $x=14 \mathrm{D}$. At this point, the inner and outer flows have been fully mixed.

\subsubsection{Acoustics in the far field}

The section above shows that the near-field turbulence from the UHBPR serrated nozzle has been well resolved. It serves as a good basis for far-field sound predictions. Figure 12 shows the near-field acoustic wave propagation. From $\partial p / \partial t$ contours, there are clearly three major sound sources. One is from the outer shear layer, another is from the inner shear layer, the third is from the interactions between the two. The near-field acoustic waves are well resolved and propagate outwards. The far-field sound pressure is predicted based on the time series of near-field FW-H surfaces.

Figure 13 shows the predictions of far-field overall sound pressure level (OASPL) from five FW-H surfaces that are placed in the near field. The placement of FW-H surfaces in Fig. 3 shows that the surface 1 is the inner most and the surface 5 is the outer most. As the surfaces are moving radially outwards, the predictions tend to show convergence, which is consistent with our previous findings in single stream 


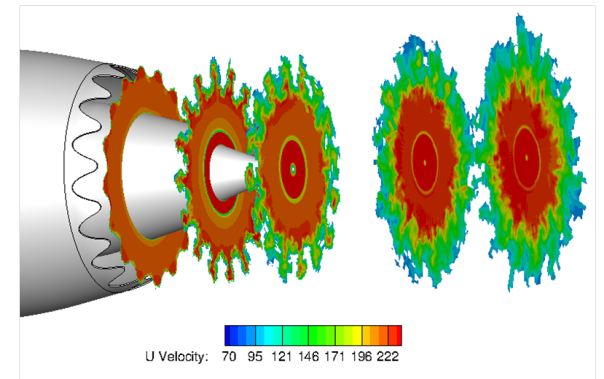

(a) Axial velocity contours

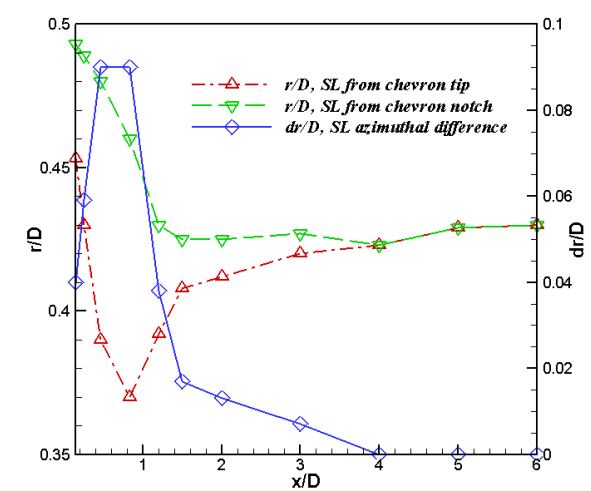

(b) shear layer radial position and azimuthal difference

Fig. 10: Shear layer development from the chevorn nozzle

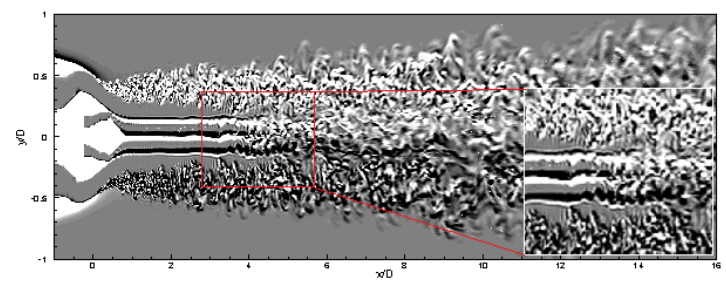

Fig. 11: Turbulence development inside the jet plume

jets [7]. The predictions from the last two surfaces(4 and 5) align with each other within $1 \mathrm{~dB}$. This convergent behaviour indicates only acoustic signals are being recorded on FWH surfaces 4 and 5

The 5th surface is used to plot the sound spectrum at different observer polar angles, shown in Fig. 14. The cut-off frequency that the mesh can support is at around $\mathrm{St}=2$. As the angle moves from higher to lower angles, the low frequency 


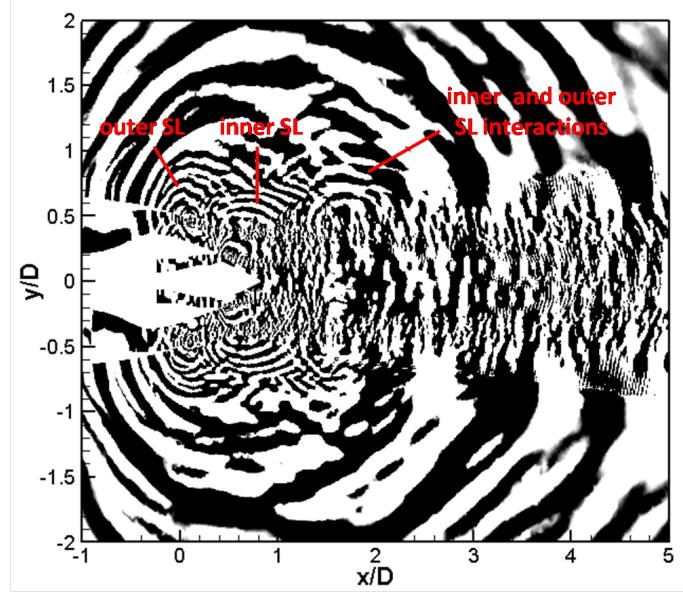

Fig. 12: Sound wave propagation in the near field, denoted by $\partial p / \partial t$

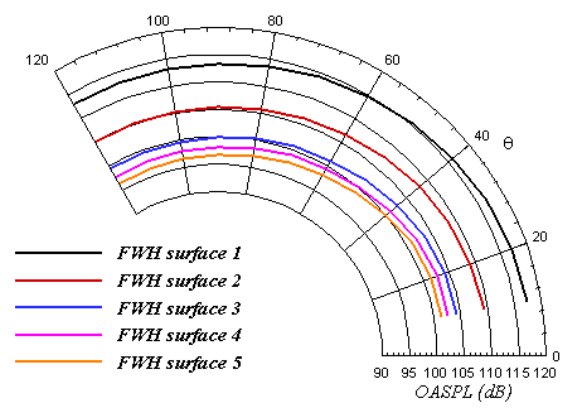

Fig. 13: Far-field OASPL predcitions

part $(S t<0.5)$ increases significantly while the high frequency part $(S t>0.5)$ rises moderately. As a result, the spectrum curves are getting steeper in the high frequency region. It indicates different sound generation mechanisms for lower and higher observer angles.

\section{Conclusions}

The capability of simulating an industrially relevant complex geometry jet has been shown in the paper. The components of this simulation methodology are demonstrated. It includes the low dissipation numerics to resolve the turbulence cascade, wall-distance based RANS/LES hybridization to treat different flow regimes, structured-unstructured mesh strategies to tackle the complex geometry and FW-H 


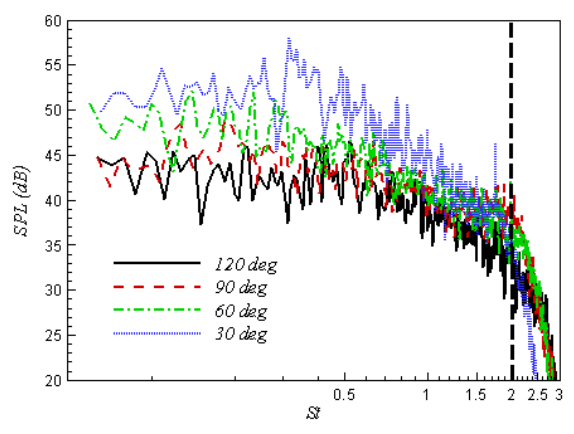

Fig. 14: Far-field sound spectra at 30, 60, 90 and 120 degree

integration methods to predict far-field sound. They have been validated on a single stream hot jet and then applied to the industrially relevant ultra-bypass serrated nozzle jet. The results show that the near-field turbulence and far-field sound are well predicted. This types of industrially complex geometry jet simulation have the potential to replace some expensive rig tests in the future and the demonstrated methodology can form a reliable industrial process chain.

Acknowledgements The authors greatly acknowledge the funds from the 7th European Framwork Program "JERONIMO" and the ARCHER computing time provided by the UK Turbulence Consortium under EPSRC grant EP/L000261/1.

\section{References}

1. Crighton, D.G., Ffowcs Williams, J.E. and Cheeseman, L.C.: The outlook for simulation of forward flight effects on aircraft noise. Journal of Aircraft. 14(11), 216-234 (2011)

2. Viswanathan, K. and Czech, M.J.: Measurement and modeling of effect of forward flight on jet noise. AIAA Journal. 49(1), 1117-1125 (1977)

3. Shur, M.L., Spalart, P.R., Srelets, M.K.: Towards the prediction of noise from jet engines. International Journal of Heat and Fluid Flow. 24(4), 551-561 (2003)

4. Bodony, D.J., Lele, S.K.: Current status of jet noise predictions using large-eddy simulation. AIAA journal. 46(2), 364-380 (2008)

5. Secundov, A.N., Birch, S.F., Tucker, P.G.: Propulsive jets and their acoustics. Philosophical Transactions of the Royal Society A: Mathematical, Physical and Engineering Sciences. 365, 2443-2467 (2007)

6. Eastwood, S., Tucker, P., Xia, H., Duckley, P. and Carpenter, P.: Large-Eddy Simulations and Measurements of a Small-Sclae High-Speed Coflowing Jet. AIAA journal. 48(5), 963-974 (2010)

7. Wang, Z.N., Naqavi, I.Z., Mahak, M., Tucker, P.G., Yuan, X. and Strange, P: Far field noise prediction of subsonic hot and cold jets using LES. ASME Turbo Expo 2014. GT2014-25928 (2014)

8. Tucker, P.G.: Differential equation-based wall distance computation for DES and RANS. Journal of Computational Physics. 190(1), 229-248 (2003) 
9. Domaradzki, J.A. and Holm, D.D.: Navier?Stokes-alpha model: LES equations with nonlinear dispersion. In:Geurts, B.J., (eds.) Modern simulation strategies for turbulent flow. Edwards Publishing, 107. ERCOFTAC Bulletin, 48(2).(2001)

10. Liu, Y., Tucker, P.G. and Kerr, R.M.: Linear and nonlinear model large-eddy simulations of a plane jet. Computers \& fluids. 37(4), 439-449 (2008)

11. Spalart, P.R. and Allmaras, S.R.: A One-Equation Turbulence Model for Aerodynamic Flows. Recherche Aerospatiale. 1, 5-21 (1994)

12. Williams, J.F. and Hawkings, D.L.: Sound generation by turbulence and surfaces in arbitrary motion. Philosophical Transactions of the Royal Society of London. Series A, Mathematical and Physical Sciences. 264(1151), 321-342 (1969)

13. Najafi-Yazdi, A., Brs, G.A. and Mongeau, L.: An acoustic analogy formulation for moving sources in uniformly moving media. Philosophical Transactions of the Royal Society of London. Series A, Mathematical and Physical Sciences. 467), 144-165 (2011)

14. Tyacke, J., Naqavi, I., Wang, Z.-N., Tucker, P. and Boehning, P.: Predictive LES for Jet Aeroacoustics - Current Approach and Industrial Application. ASME Turbo Expo 2016. GT201656673 (2016)

15. Mockett, C., Fuchs, M., Kramer, F., Michel, U., Thiele, F. and Steger, M.: Turbulence Modelling and Meshing Developments for the Prediction of Jet Noise Installation Effects. AIAA2016-2933 (2016)

16. Bridges, J and Wernet, M.P.: Establishing Consensus Turbulence Statistics for Hot Subsonic Jets. AIAA2010-3751 (2010) 